\title{
ESCUELA MODERNA, ALUMNOS QUE APRENDEN
}

Modern School, students who learn

Escola Moderna, estudantes que aprendem

\section{Pablo Casado Berrocal}

CEIP “Villa y Tierra” de Saldaña (Palencia), España. Teléfono +34 660821826. Correo electrónico: pablocasado5@gmail.com

\section{Resumen}

La experiencia presenta los resultados obtenidos en el proceso de renovación pedagógica puesto en práctica en una escuela rural. Durante un curso académico se utilizaron técnicas y recursos relacionados con la Escuela Moderna. En el texto se describe un proceso de modernización escolar no intrusivo mediante sencillas técnicas (plan de trabajo, fichero escolar cooperativo, fichero autocorrectivo, etc.) que permiten la convivencia saludable con otros métodos y técnicas propios de cualquier variante o movimiento pedagógico que tenga como objetivo la democratización de la enseñanza y de los procesos de evaluación. Los resultados obtenidos ponen de manifiesto la importancia que poseen las decisiones metodológicas y de evaluación en los procesos de aprendizaje y la posibilidad innegable que poseemos los docentes para aprovechar la predisposición innata que el ser humano muestra hacia su aprendizaje.

Palabras clave: Escuela Moderna; evaluación formativa; metodologías activas; proyectos; autorregulación

\begin{abstract}
The experience presents the results obtained in the pedagogical renewal process put into practice in a rural school. During an academic year, techniques and resources related to the Modern School were used. The text describes a process of non-intrusive school modernization that allows healthy coexistence with other methods and techniques specific to any variant or pedagogical movement aimed at democratizing teaching and assessment processes. The results show the importance of the methodological and assessment decisions in the learning processes and the undeniable possibility that teachers have to take advantage of the innate predisposition that the human being shows towards their learning.
\end{abstract}


Keywords: Modern School; Formative assessment; Active methodologies; Projects; self-regulation

\section{Resumo}

Experiência apresenta os resultados obtidos no processo de reforma educativa implementada em uma escola rural. Durante um ano, e técnicas relacionadas com os recursos escola moderna acadêmica eles foram usados. No texto, um processo de modernização escolar que permite convivência saudável não-intrusiva com outros métodos e técnicas de qualquer variante ou movimento educacional que visa democratizar os processos de ensino e de avaliação descritos próprios. Os resultados mostram a importância de ter os processos de avaliação e decisão metodológicas de aprendizagem e possibilidade inegável que os professores possuem a predisposição inata para tirar vantagem de que o homem mostra a sua aprendizagem.

Palavras-chave: Escola Moderna; avaliação formativa; metodologias activas; projetos; auto-regulação

\section{Introducción}

Desde hace más de un siglo no son pocos los docentes que identifican la modernización de la escuela como cimiento esencial en la mejora de la calidad educativa, proceso sobre el que pretendía poner el énfasis la Ley Orgánica 8/2013, de 9 de diciembre, para la Mejora de la Calidad Educativa.

Sin embargo, conseguir una Escuela Moderna sigue siendo a día de hoy un logro no alcanzado. En los últimos años asistimos a un constante goteo de noticias, artículos, gurús y mesías de la “panacea educativa” que solo han supuesto mejoras superficiales. En muchas ocasiones, los instrumentos, las técnicas y el método no han cambiado en absoluto: lecciones, deberes, estudio de textos, las notas, los castigos y el supremo recurso a la memoria como llave de acceso a una "evaluación” que solo tiene como objetivo la calificación del alumno (Freinet y Salengros, 1976).

En la experiencia que se recoge en este texto se presentan algunos procesos, técnicas y recursos válidos para llevar a cabo una renovación pedagógica de transición que no rompe drásticamente con otros métodos y técnicas, sino que convive con ellos y los hace perfectamente viables en la realidad educativa, poniendo el énfasis en la 
importancia que tiene democratizar los procesos de enseñanza y de evaluación que ocurren diariamente.

\section{Contextualización}

La experiencia muestra un proceso de modernización escolar centrado en el área de Ciencias de la Naturaleza en un CEIP rural de doble línea que cuenta con 436 alumnos. En ella, han participado un total de 51 alumnos de $6^{\circ}$ de Educación Primaria divididos en dos grupos (A y B).

Durante un curso académico, se pusieron en práctica las técnicas descritas más adelante con una frecuencia de, al menos, 2,5h/semana.

Ambos grupos habían permanecido durante tres cursos académicos con sus tutores actuales. Estos habían puesto en práctica de manera puntual estrategias vinculadas al Aprendizaje Cooperativo (Zariquiey, 2015; Pujolás, 2011; Johnson \& Johnson, 1999).

\section{Diseño}

La fase de diseño de la experiencia se desarrolla durante el primer mes del curso académico (septiembre), llevándose a cabo los siguientes procesos:

1. Cuestionario individual realizado a cada alumno sobre la forma de trabajo en el área en cursos anteriores (información y valoración).

2. Entrevista informativa con los docentes responsables del área en el curso anterior.

3. Listado de propuestas de mejora aportadas por los grupos de alumnos y el docente actual.

4. Selección de técnicas y procesos necesarios con los que se pretendía conseguir una mejora.

5. Secuenciación de técnicas y procesos de forma dialogada con los grupos de alumnos.

\section{Desarrollo}

La fase de desarrollo de la experiencia comprende los meses de octubre a junio, y en ella se introdujeron de forma sistemática las siguientes mejoras relacionadas con procesos de autorregulación (Zimmerman \& Schunk, 1990). 
Tabla 1.

Secuenciación de procesos vinculados al desarrollo de la experiencia

\begin{tabular}{|c|c|}
\hline PROCESO & TEMPORALIZACIÓN \\
\hline Plan de trabajo como técnica de planificación personal & \multirow{4}{*}{ Primer trimestre } \\
\hline Instrumentos sencillos de heteroevaluación final (docente a alumno) & \\
\hline Instrumentos sencillos de autoevaluación (alumno) & \\
\hline $\begin{array}{l}\text { Solucionario como instrumento para el fomento de la autonomía en la } \\
\text { corrección de tareas }\end{array}$ & \\
\hline $\begin{array}{l}\text { Talleres como técnica para la sustitución parcial de ejercicios } \\
\text { mecánicos }\end{array}$ & \multirow{4}{*}{ Segundo trimestre } \\
\hline Instrumentos sencillos de coevaluación intragrupal (alumno a alumno) & \\
\hline $\begin{array}{l}\begin{array}{l}\text { Instrumentos sencillos de heteroevaluación formativa y continua } \\
\text { (docente a alumno) }\end{array} \\
\end{array}$ & \\
\hline $\begin{array}{l}\text { Fichero cooperativo del grupo como técnica para la sustitución parcial } \\
\text { de ejercicios mecánicos }\end{array}$ & \\
\hline $\begin{array}{l}\text { Conferencia/presentación como procedimiento de evaluación en } \\
\text { sustitución del examen }\end{array}$ & \multirow{5}{*}{ Tercer trimestre } \\
\hline Instrumentos sencillos de coevaluación grupal (grupo a grupo) & \\
\hline Instrumentos sencillos de heteroevaluación (alumno a docente) & \\
\hline Preguntas generadoras como punto de partida para el aprendizaje & \\
\hline Proyecto grupal como actividad de consolidación y puesta en práctica & \\
\hline
\end{tabular}

Esta fase de desarrollo permitió vislumbrar algunos resultados iniciales que se detallan más adelante en el apartado “conclusiones”; destacando especialmente la mejora de la autonomía del alumnado en los procesos vinculados a su aprendizaje y la mejora de su disposición hacia la temática del área

\section{Evaluación}

La evaluación de la experiencia global y de las técnicas puestas en práctica se realiza de forma sistemática a través de los siguientes procesos (Tabla 2). El proceso relativo a la comparación de resultados iniciales (septiembre) y finales (junio) puso de manifiesto la progresión mostrada por la mayor parte del grupo durante el curso escolar en el área de Ciencias de la Naturaleza.

Tabla 2.

Secuenciación de procesos vinculados a la evaluación de la experiencia

\begin{tabular}{lcc}
\hline \multicolumn{1}{c}{ PROCESO } & TEMPORALIZACIÓN \\
\hline Prueba de evaluación inicial de conocimientos del alumnado & Primer trimestre \\
\hline $\begin{array}{l}\text { Cuestionario para el alumno sobre la valoración del área de Ciencias } \\
\text { de la Naturaleza (contenidos, procesos, actividades, etc.) }\end{array}$ & Trimestralmente \\
\hline $\begin{array}{l}\text { Registro anecdótico sobre los procesos de aprendizaje en el aula y } \\
\text { redacción de conclusiones }\end{array}$ & \multirow{2}{\text{Usodelbuzóndesugerenciasanónimasybuzóndeagradecimientos}}{$\begin{array}{c}\text { Durante todo el curso } \\
\text { académico }\end{array}$} \\
$\begin{array}{l}\text { Comparación de resultados/calificaciones del alumnado (progresión } \\
\text { durante el trimestre y respecto al curso anterior) }\end{array}$ & \\
\hline Entrevistas informales con las familias de los alumnos de cada grupo & \\
\hline
\end{tabular}




\section{Conclusiones}

El desarrollo de la experiencia recogida en el texto arroja resultados concluyentes y muy positivos en diversas categorías: calificaciones del alumnado, interés y gusto por el área, autonomía, expresión escrita, expresión oral, actitud del alumnado en el entorno familiar, etc.

Estos resultados ponen el acento en la importancia que poseen las decisiones metodológicas y de evaluación en los procesos de aprendizaje y la posibilidad innegable que poseemos los docentes para aprovechar la predisposición innata que el ser humano muestra hacia su aprendizaje.

Con los aspectos incluidos en este documento se ha intentado constituir el punto de partida para un nuevo horizonte, una nueva línea de investigación-acción que sirva de marco común para establecer las posibles fases de un modelo de transición necesario para modernizar la escuela a través del uso de diferentes estrategias metodológicas y de evaluación.

\section{Referencias}

Freinet, C. (2005). Técnicas Freinet para la Escuela Moderna. Madrid: Siglo XXI.

Freinet, C., \& Salengros, R. (1976). Modernizar la escuela. Barcelona: Laia.

Johnson, D. \& Johnson, R. (1999). El aprendizaje cooperativo en el aula. Buenos Aires: Paidós.

Pujolás, P. (2011). El aprendizaje cooperativo. Nueve ideas clave. Barcelona: Graó.

Zariquiey, F. (2015). Guía para diseñar y poner en marcha una red de aprendizaje cooperativo. Madrid: Colectivo Cinética.

Zimmerman, B.J., \& Schunk, D.H. (1990). Self-regulated learning and academic achievement. Educational Psychologist, 25(1), 3-17. 